\title{
Antimicrobial Resistance in Respiratory Pathogens Isolated in Brazil During 1999-2000
}

Ian A. Critchley, Renée S. Blosser, James A. Karlowsky, Juri Yamakita, Alfonso Barth, Helio S. Sader, Caio Mendes, Lucia Teixeira, Flavia Rossi, Cicero A. C. Dias, Mark E. Jones, Clyde Thornsberry and Daniel F. Sahm
Focus Technologies, Inc. (formerly MRL), Herndon, Virginia, USA (IAC, RSB, JAK, DFS); Daiichi

Pharmaceutical Co., Ltd., Chuo-Ku, Tokyo, Japan (JY); Hospital de Clinicas de Porto Alegre, Porto Alegre, Brazil (AB); Universidade Federal de São Paulo, Sao Paulo, Brazil (HSS); Laboratorio Fleury, Sao Paulo, Brazil (CM); Federal University of Rio de Janeiro, Rio de Janeiro, Brazil (LT); Hospital das Clínicas, FMUSP, Sao Paulo, Brazil (FR); Fundacao Fuculdade de Ciencias, Porto Alegre, Brazil (CACD); Focus Technologies, Inc. (formerly MRL), Hilversum, The Netherlands (MEJ), and Brentwood, Tennessee, USA (CT)

The in vitro antimicrobial susceptibility of the respiratory pathogens Streptococcus pneumoniae, Haemophilus influenzae, and Moraxella catarrhalis to commonly tested and prescribed agents was investigated during 1999-2000 and compared with results obtained during a previous 1997-1998 study. Of 448 isolates of $S$. pneumoniae collected and tested in 1999-2000, 77.2\% were susceptible, $19.9 \%$ were intermediate, and $2.9 \%$ were resistant to penicillin, demonstrating that there were no major changes in susceptibility to penicillin from 1997-1998 (77.1\% susceptible, 18.7\% intermediate, 4.2\% resistant). All $S$. pneumoniae isolates from 1999-2000 were susceptible to levofloxacin and vancomycin, and $>90 \%$ were susceptible to the $\beta$-lactams (amoxicillin-clavulanate, ceftriaxone, and cefuroxime) and macrolides (azithromycin and clarithromycin), showing that susceptibility to these agents also remained unchanged since 1997-1998. The most notable increase in resistance between the two studies was demonstrated by trimethoprim-sulfamethoxazole, which increased from $23.4 \%$ to $38.6 \%$. Penicillin resistance correlated with resistance to $\beta$-lactams, macrolides, and trimethoprimsulfamethoxazole in both studies. In $\mathrm{H}$. influenzae, the prevalence of $\beta$-lactamase-producing isolates remained unchanged $(10.6 \%$ in $1999-2000 ; 11.0 \%$ in 1997-1998). All $H$. influenzae isolates were susceptible to levofloxacin, ceftriaxone, cefuroxime, and azithromycin, and showed no change between the two studies. Trimethoprim-sulfamethoxazole resistance was present in $40.1 \%$ of isolates in 19992000, and in 45.2\% in 1997-1998. In M. catarrhalis, the prevalence of $\beta$-lactamase-producing isolates was unchanged $(97.9 \%$ in $1999-2000 ; 98.0 \%$ in $1997-1998)$. The most active agents against $M$. catarrhalis were azithromycin ( $\mathrm{MIC}_{90}, \leq 0.03 \mu \mathrm{g} / \mathrm{ml}$ ) and levofloxacin $\left(\mathrm{MIC}_{90}, 0.03 \mu \mathrm{g} / \mathrm{ml}\right.$ ). Overall, these results suggest that, in Brazil, between 1999-2000 and 1997-1998, there have been no significant changes in the susceptibility of respiratory pathogens to any of the commonly tested and prescribed agents with the exception of trimethoprim-sulfamethoxazole for S. pneumoniae.

KeyWords: Streptococcus pneumoniae, antimicrobial resistance, surveillance study, respiratory pathogen.

Received on 25 July 2001; revised 24 October 2001.

Address for correspondence: Focus Technologies, Inc. (formerly MRL), 13665 Dulles Technology Drive, Suite 200, Herndon, VA 20171-4603. Phone: (703) 480-2535. Fax: (703) 480-2654.E-mail: icritchley@mrlinfo.com.

The Brazilian Journal of Infectious Diseases 2001;5(6):294-304 . (C) 2001 by The Brazilian Journal of Infectious Diseases and Contexto Publishing. All rights reserved.

1413-8670
The respiratory pathogens Streptococcus pneumoniae, Haemophilus influenzae, and Moraxella catarrhalis have generated considerable interest and a number of worldwide surveillance initiatives has demonstrated emerging resistance to different classes of antimicrobial agents. $S$. pneumoniae isolates with reduced susceptibility to penicillin were first reported in 1967 [1], and were 
subsequently detected in Europe, South Africa, and Japan in the 1970s [2], and in Brazil in 1988 [3]. Of particular concern is the tendency for penicillin-resistant pneumococci to exhibit reduced susceptibility to other agents, including other $\beta$-lactams, macrolides, and trimethoprim-sulfamethoxazole [4-8]. Annual surveillance of resistance in respiratory pathogens is now being conducted in the United States and has shown that significant changes in antimicrobial resistance profiles can occur between two sequential respiratory seasons [8]. For example, trimethoprimsulfamethoxazole exhibited a two-fold increase in resistance for S. pneumoniae between the 1997-1998 and 1998-1999 respiratory seasons [8]. Although fluoroquinolone-resistant isolates of pneumococci have been reported, they are still relatively rare $(\leq 1 \%$ resistance) [8,9].

The acquisition of plasmid-encoded $\beta$-lactamases in isolates of Haemophilus influenzae and Moraxella catarrhalis has largely eliminated ampicillin as a therapeutic agent for infections caused by $\beta$-lactamaseproducing isolates $[10,11]$. Resistance to trimethoprimsulfamethoxazole in $H$. influenzae is also prevalent in many countries $[7,10]$. To date, the fluoroquinolones have retained their activity against $H$. influenzae and M. catarrhalis in most countries, including Brazil. It is clear that continuous surveillance efforts are vital in monitoring any changes in the susceptibility profiles of respiratory pathogens.

We report the results of a study intended to monitor antimicrobial resistance in respiratory pathogens in Brazil during the 1999-2000 respiratory season. Since the isolates were collected from the same hospital laboratories that participated in a previous study we conducted in 1997-1998 [12], we were also able to perform a longitudinal analysis of the results to identify any significant changes in antimicrobial resistance in Brazil during the two seasons.

\section{Materials and Methods}

Organism collection. From April 1999 to April 2000, we collected respiratory isolates from six hospital laboratories in Brazil. Each laboratory submitted one isolate per patient, and each isolate was accompanied by patient data. Five of the six laboratories also participated in the respiratory pathogen surveillance study we conducted in Brazil in 1997-1998 [12], enabling a longitudinal analysis of resulting data. All isolates were derived from clinical samples taken from upper and lower respiratory tract sites, blood, ears, and eyes. All isolates were shipped to the Focus Technologies, Inc. (formerly Focus Technologies) central laboratory, in Herndon, Virginia, USA, where each isolate was subcultured onto blood agar (or chocolate agar in the case of $H$. influenzae) and reidentified according to standard methods as previously described $[6,8,10,12]$. Pure cultures were stocked in the MRL culture collection, and all isolates were maintained frozen at $-80^{\circ} \mathrm{C}$. In total, 448 isolates of S. pneumoniae, 274 isolates of $H$. influenzae, and 49 isolates of $M$. catarrhalis were collected during 1999-2000 and tested against commonly prescribed antimicrobial agents. In the 1997-1998 study, a total of 359 isolates of $S$. pneumoniae, 219 isolates of $H$. influenzae, and 52 isolates of $M$ catarrhalis were collected from five laboratories.

Antibiotic susceptibility testing. Isolates were tested for susceptibility to penicillin (S. pneumoniae), ampicillin ( $H$. influenzae and $M$. catarrhalis), amoxicillin-clavulanate, ceftriaxone, cefuroxime, vancomycin, azithromycin, clarithromycin, trimethoprim-sulfamethoxazole, and levofloxacin using concentrations that were at least one 2-fold concentration below the susceptible breakpoint and one 2-fold concentration above the resistant breakpoint (when available). Antibiotic susceptibility testing was conducted by broth microdilution using dried Sensititre panels (Trek Diagnostic Systems, Inc., Westlake, Ohio) in accordance with NCCLS guidelines. For $S$. pneumoniae and $H$. influenzae, breakpoint interpretations were conducted according to the recommendations of the National Committee for Clinical Laboratory Standards (NCCLS) [13,14]. For M. catarrhalis, no NCCLS breakpoints were available. All H. influenzae and M. catarrhalis isolates 
were tested for the production of $\beta$-lactamase using the DrySlide nitrocefin test (Difco Laboratories, Detroit, Michigan).

\section{Results}

S. pneumoniae. Table 1 shows the antimicrobial activity of all antimicrobials tested against $S$. pneumoniae collected during the 1999-2000 respiratory season in Brazil. Of the 448 isolates collected and tested, 89 (19.9\%) were intermediate and $13(2.9 \%)$ were resistant to penicillin. Based on $\mathrm{MIC}_{90} \mathrm{~s}$, amoxicillin-clavulanate was the most potent $\beta$-lactam $\left(\mathrm{MIC}_{90}, 0.06 \mu \mathrm{g} / \mathrm{ml} ; 0.9 \%\right.$ resistant), with only four resistant isolates, closely followed by ceftriaxone $\left(\mathrm{MIC}_{90}, 0.12 \mu \mathrm{g} / \mathrm{ml} ; 0.4 \%\right.$ resistant), with only two resistant isolates. Cefuroxime was the least active $\beta$-lactam ( $\mathrm{MIC}_{90}, 0.5 \mu \mathrm{g} / \mathrm{ml} ; 5.4 \%$ resistant), with 24 isolates resistant. Penicillin is a marker antimicrobial often used to stratify the activity of other antimicrobial agents against $S$. pneumoniae. Among the $\beta$-lactams (amoxicillin-clavulanate, ceftriaxone, and cefuroxime) there was a correlation with susceptibility to penicillin, as all resistant isolates were nonsusceptible (intermediate or resistant) to penicillin. None of the isolates collected in the 1999-2000 season were intermediate or resistant to levofloxacin. The activity of levofloxacin was unaffected by susceptibility to penicillin, as penicillin-susceptible, -intermediate, and -resistant isolates all had $\mathrm{MIC}_{90} \mathrm{~s}$ of $1 \mu \mathrm{g} / \mathrm{ml}$.

Azithromycin and clarithromycin demonstrated equivalent antibacterial activity against all isolates, with $\mathrm{MIC}_{90} \mathrm{~s}$ of 0.06 and $0.03 \mu \mathrm{g} / \mathrm{ml}$ and resistance rates of $4.7 \%$ and $4.9 \%$, respectively. Resistance rates to azithromycin (30.8\%) and clarithromycin (38.5\%) were higher in isolates that were resistant to penicillin. Trimethoprim-sulfamethoxazole was the least active agent $\left(\mathrm{MIC}_{90},>4 \mu \mathrm{g} / \mathrm{ml}\right.$; resistance rate, $\left.38.6 \%\right)$ and also showed cross-resistance to penicillin, with all 13 penicillin-resistant isolates also resistant to trimethoprim-sulfamethoxazole.

All 448 isolates of S. pneumoniae were analyzed for the presence of multidrug resistance, defined as resistance to three or more classes of antimicrobial agents. We identified six multidrug-resistant (MDR) isolates, a prevalence of $1.3 \%$. Four of the MDR isolates were resistant to azithromycin, penicillin, and trimethoprim-sulfamethoxazole, and two were resistant to ceftriaxone, penicillin, and trimethoprimsulfamethoxazole (data not shown).

Specimen source and patient age data were collected for each isolate and were analyzed for possible associations with antimicrobial susceptibility. In $S$. pneumoniae, penicillin resistance increased from $1.0 \%$ in isolates from patients $>18$ years of age to $5.0 \%$ in isolates from patients $>65$ years old. Macrolide resistance also varied with patient age; resistance rates in patients $<18$ years old were $2.6 \%$ for clarithromycin and $3.1 \%$ for azithromycin, compared to $10.0 \%$ resistance for both agents in patients $>65$ years old. Analysis of results by specimen source showed that penicillin resistance in $S$. pneumoniae ranged from $2.2 \%$ in respiratory isolates to $5.6 \%$ in blood isolates (data not shown).

Changes in the resistance of $S$. pneumoniae to all tested antimicrobials between the study we conducted in 1997-1998 [12] and the current 1999-2000 study are shown in Table 2. For penicillin, the percentage of nonsusceptible isolates remained unchanged between the 2 years; however, there was a minor shift in MIC distribution from the intermediate to the resistant category in the 1999-2000 study (18.7\% intermediate; $4.2 \%$ resistant) compared with the 1997-1998 study (19.9\% intermediate; $2.9 \%$ resistant). Amoxicillinclavulanate, cefuroxime, and ceftriaxone demonstrated only minor changes in the percentages of resistant isolates $(<1 \%)$, as did the macrolides azithromycin and clarithromycin $(<0.5 \%)$. The percentage of isolates susceptible to vancomycin remained at $100 \%$, and there was no change in the $\mathrm{MIC}_{90}(0.5 \mu \mathrm{g} / \mathrm{ml})$. Susceptibility to levofloxacin also remained stable, with no intermediate or resistant isolates reported in the 19992000 study, and one resistant isolate reported in the 1997-1998 study. The largest increase in resistance was demonstrated by trimethoprim-sulfamethoxazole, with $23.4 \%$ resistance in $1997-1998$, and $38.6 \%$ in 1999-2000. Further analysis showed that the 
Table 1. Susceptibility of 448 isolates of S. pneumoniae collected during 1999-2000 to all antimicrobials tested according to penicillin status ${ }^{\mathrm{a}}$

\begin{tabular}{|c|c|c|c|c|c|c|c|}
\hline & \multicolumn{3}{|c|}{$\mu \mathbf{g} / \mathbf{m l}$} & \multirow[b]{2}{*}{ Mode } & \multirow[b]{2}{*}{$\% \mathrm{~S}$} & \multirow[b]{2}{*}{$\%$ I } & \multirow[b]{2}{*}{$\% R$} \\
\hline & Range & $\mathrm{MIC}_{50}$ & $\mathrm{MIC}_{90}$ & & & & \\
\hline Pencillin & $\leq 0.03-4$ & $\leq 0.03$ & 0.25 & $\leq 0.03$ & 77.2 & 19.9 & 2.9 \\
\hline Penicillin-susceptible & $\leq 0.03-0.06$ & $\leq 0.03$ & $\leq 0.03$ & $\leq 0.03$ & 100 & 0 & 0 \\
\hline Penicillin-intermediate & $0.12-1$ & 0.12 & 1 & 0.12 & 0 & 100 & 0 \\
\hline Penicillin-resistant & $2-4$ & 2 & 2 & 2 & 0 & 0 & 100 \\
\hline Amoxicillin-clavulanate & $\leq 0.015-2$ & 0.03 & 0.06 & $\leq 0.015$ & 96.9 & 2.2 & 0.9 \\
\hline Penicillin-susceptible & $\leq 0.015-0.12$ & $\leq 0.015$ & 0.03 & $\leq 0.015$ & 100 & 0 & 0 \\
\hline Penicillin-intermediate & $0.03-1$ & 0.06 & 0.5 & 0.06 & 96.6 & 3.4 & 0 \\
\hline Penicillin-resistant & $0.5-2$ & 1 & 2 & 1 & 15.4 & 53.8 & 30.8 \\
\hline Ceftriaxone & $\leq 0.015-2$ & 0.03 & 0.12 & 0.03 & 97.1 & 2.5 & 0.4 \\
\hline Penicillin-susceptible & $\leq 0.015-0.25$ & 0.03 & 0.06 & 0.03 & 100 & 0 & 0 \\
\hline Penicillin-intermediate & $\leq 0.015-1$ & 0.12 & 0.5 & 0.12 & 98.9 & 1.1 & 0 \\
\hline Penicillin-resistant & $0.5-2$ & 1 & 2 & 1 & 7.7 & 76.9 & 15.4 \\
\hline Cefuroxime & $\leq 0.12-8$ & $\leq 0.12$ & 0.5 & $\leq 0.12$ & 91.5 & 3.1 & 5.4 \\
\hline Penicillin-susceptible & $\leq 0.12-1$ & $\leq 0.12$ & $\leq 0.12$ & $\leq 0.12$ & 99.1 & 0.9 & 0 \\
\hline Penicillin-intermediate & $\leq 0.12-4$ & 0.5 & 2 & 0.5 & 75.3 & 12.4 & 12.4 \\
\hline Penicillin-resistant & $4-8$ & 4 & 8 & 4 & 0 & 0 & 100 \\
\hline Azithromycin & $\leq 0.03->4$ & $\leq 0.03$ & 0.06 & $\leq 0.03$ & 94.9 & 0.4 & 4.7 \\
\hline Penicillin-susceptible & $\leq 0.03->4$ & $\leq 0.03$ & $\leq 0.03$ & $\leq 0.03$ & 96.8 & 0 & 3.2 \\
\hline Penicillin-intermediate & $\leq 0.03->4$ & $\leq 0.03$ & 0.12 & $\leq 0.03$ & 92.1 & 1.1 & 6.7 \\
\hline Penicillin-resistant & $\leq 0.03->4$ & $\leq 0.03$ & $>4$ & $\leq 0.03$ & 61.5 & 7.7 & 30.8 \\
\hline Clarithromycin & $\leq 0.015->32$ & 0.03 & 0.03 & $\leq 0.015$ & 94.9 & 0.2 & 4.9 \\
\hline Penicillin-susceptible & $\leq 0.015->32$ & $\leq 0.015$ & 0.03 & $\leq 0.015$ & 96.8 & 0 & 3.2 \\
\hline Penicillin-intermediate & $\leq 0.015->32$ & 0.03 & 0.03 & 0.03 & 92.1 & 1.1 & 6.7 \\
\hline Penicillin-resistant & $\leq 0.015->32$ & 0.03 & $>32$ & $\leq 0.015$ & 61.5 & 0 & 38.5 \\
\hline TMP-SMX & $\leq 0.015->4$ & 1 & $>4$ & 4 & 44.9 & 16.5 & 38.6 \\
\hline Penicillin-susceptible & $\leq 0.015->4$ & 0.5 & 4 & 0.25 & 55.5 & 16.5 & 28.0 \\
\hline Penicillin-intermediate & $0.12->4$ & 4 & $>4$ & 4 & 10.1 & 19.1 & 70.8 \\
\hline Penicillin-resistant & $4->4$ & $>4$ & $>4$ & $>4$ & 0 & 0 & 100 \\
\hline Vancomycin & $\leq 0.06-0.5$ & 0.25 & 0.5 & 0.25 & 100 & $-c$ & - \\
\hline Penicillin-susceptible & $\leq 0.06-0.5$ & 0.25 & 0.5 & 0.25 & 100 & - & - \\
\hline Penicillin-intermediate & $0.12-0.5$ & 0.25 & 0.5 & 0.25 & 100 & - & - \\
\hline Penicillin-resistant & $0.25-0.5$ & 0.25 & 0.5 & 0.25 & 100 & - & - \\
\hline Levofloxacin & $0.25-2$ & 1 & 1 & 1 & 100 & 0 & 0 \\
\hline Penicillin-susceptible & $0.25-1$ & 1 & 1 & 1 & 100 & 0 & 0 \\
\hline Penicillin-intermediate & $0.5-2$ & 1 & 1 & 1 & 100 & 0 & 0 \\
\hline Penicillin-resistant & $0.5-1$ & 1 & 1 & 1 & 100 & 0 & 0 \\
\hline
\end{tabular}

a346 Penicillin-susceptible, 89 penicillin-intermediate, and 13 penicillin-resistant $S$. pneumoniae were collected.

${ }^{\mathrm{b}} \mathrm{TMP}-\mathrm{SMX}=$ trimethoprim-sulfamethoxazole.

${ }^{\mathrm{N}} \mathrm{NCCLS}$ breakpoints were unavailable for categorical interpretation as intermediate (I), or resistant (R). 
Table 2. Comparison of resistance prevalence and $\mathrm{MIC}_{90} \mathrm{~s}$ for respiratory pathogens collected in the 1997-1998 and 1999-2000 respiratory seasons

\begin{tabular}{|c|c|c|c|c|c|c|c|c|}
\hline & \multirow[t]{2}{*}{ Year } & \multicolumn{3}{|c|}{ S. pneumoniae } & \multicolumn{3}{|c|}{ H. influenzae } & \multirow{2}{*}{$\frac{\text { M. catarrhalis }}{\mathrm{MIC}_{90}}$} \\
\hline & & $\mathrm{MIC}_{90}$ & $\% \mathbf{I}$ & $\% R$ & $\mathrm{MIC}_{90}$ & $\%$ I & $\% \mathbf{R}$ & \\
\hline \multirow{2}{*}{ Penicillin } & 1997-1998 & 0.25 & 18.7 & 4.2 & $\mathrm{NT}^{\mathrm{d}}$ & NT & NT & NT \\
\hline & 1999-2000 & 0.25 & 19.9 & 2.9 & NT & NT & NT & NT \\
\hline \multirow{2}{*}{ Ampicillin } & 1997-1998 & NT & NT & NT & 2 & 1.4 & 9.6 & $>8$ \\
\hline & 1999-2000 & NT & NT & NT & 8 & 0.4 & 10.6 & \\
\hline \multirow[t]{2}{*}{ Amoxicillin-clavulanate } & $1997-1998^{a}$ & 0.06 & 2.2 & 1.7 & 1 & 0 & 0 & 0.25 \\
\hline & $1999-2000^{b}$ & 0.06 & 2.2 & 0.9 & 1 & 0 & 0.4 & 0.25 \\
\hline \multirow[t]{2}{*}{ Ceftriaxone } & 1997-1998 & 0.12 & 2.8 & 1.4 & 0.03 & 0 & 0 & 0.5 \\
\hline & 1999-2000 & 0.12 & 2.5 & 0.4 & $\leq 0.015$ & 0 & 0 & 0.5 \\
\hline \multirow[t]{2}{*}{ Cefuroxime } & 1997-1998 & 0.5 & 2.2 & 5.3 & 2 & 0 & 0 & 2 \\
\hline & 1999-2000 & 0.5 & 3.1 & 5.4 & 1 & 0 & 0 & 2 \\
\hline \multirow{2}{*}{ Azithromycin } & 1997-1998 & 0.06 & 0.6 & 4.2 & 2 & $-^{\mathrm{c}}$ & - & $\leq 0.03$ \\
\hline & 1999-2000 & 0.06 & 0.4 & 4.7 & 2 & - & - & $\leq 0.03$ \\
\hline \multirow[t]{2}{*}{ Clarithromycin } & 1997-1998 & 0.03 & 0 & 4.7 & 8 & 7.8 & 0 & 0.12 \\
\hline & 1999-2000 & 0.03 & 0.2 & 4.9 & 8 & 2.6 & 0.4 & 0.12 \\
\hline \multirow[t]{2}{*}{ TMP-SMX } & 1997-1998 & 4 & 28.4 & 23.4 & $>4$ & 7.8 & 45.2 & 0.5 \\
\hline & 1999-2000 & $>4$ & 16.5 & 38.6 & $>4$ & 9.5 & 40.1 & 0.5 \\
\hline \multirow[t]{2}{*}{ Vancomycin } & 1997-1998 & 0.5 & - & - & NT & $\mathrm{NT}$ & NT & NT \\
\hline & 1999-2000 & 0.5 & - & - & NT & NT & NT & NT \\
\hline \multirow[t]{2}{*}{ Levofloxacin } & 1997-1998 & 1 & 0 & 0.3 & 0.015 & 0 & 0 & 0.06 \\
\hline & 1999-2000 & 1 & 0 & 0 & 0.015 & 0 & 0 & 0.03 \\
\hline
\end{tabular}

a359 S. pneumoniae, 219 H. influenzae, and 52 M. catarrhalis were collected during 1997-1998.

b448 S. pneumoniae, 274 H. influenzae, and 49 M. catarrhalis were collected during 1999-2000.

'NCCLS breakpoints unavailable for categorical interpretation as susceptible (S), intermediate (I), or resistant (R).

${ }^{\mathrm{d}} \mathrm{NT}=$ not tested.

eTMP-SMX = trimethoprim-sulfamethoxazole.

${ }_{\mathrm{f}}$ NCCLS breakpoints unavailable for categorical interpretation as intermediate (I) or resistant (R). 
trimethoprim-sulfamethoxazole-resistant isolates were evenly distributed among the participating laboratories in both studies (data not shown).

H. influenzae. The results in Table 3 show the activity of antimicrobial agents against the 274 isolates of $H$. influenzae collected during the 1999-2000 study. Of the 274 isolates, $29(10.6 \%)$ produced $\beta$-lactamase, and 28 of those were also ampicillin-resistant. One isolate that was $\beta$-lactamase-negative and ampicillinresistant (BLNAR) originated from a 4-year-old male outpatient and was also resistant to amoxicillinclavulanate. Ceftriaxone was the most active $\beta$-lactam based on $\mathrm{MIC}_{90}(\leq 0.015 \mu \mathrm{g} / \mathrm{ml})$ and its activity was not affected by the production of $\beta$-lactamase. Azithromycin ( $\mathrm{MIC}_{90}, 2 \mu \mathrm{g} / \mathrm{ml}$; susceptibility 100\%) was four-fold more potent than clarithromycin $\left(\mathrm{MIC}_{90}\right.$, $8 \mu \mathrm{g} / \mathrm{ml}$; susceptibility 97.1\%). Trimethoprimsulfamethoxazole was the least active of the agents tested, with an $\mathrm{MIC}_{90}$ of $>4 \mu \mathrm{g} / \mathrm{ml}$ and a resistance rate of $40.1 \%$. Furthermore, the activity of trimethoprim-sulfamethoxazole was adversely affected by the production of $\beta$-lactamase, with $34.7 \%$ of $\beta$ lactamase-nonproducing isolates resistant, compared with $86.2 \%$ of $\beta$-lactamase-producing isolates. The 110 trimethoprim-sulfamethoxazole-resistant isolates of $H$. influenzae were evenly distributed across the six laboratories, with resistance rates ranging from $23.5 \%$ to $55.4 \%$ (data not shown). The results in Table 2 show that there were no major changes in the susceptibility of $H$. influenzae to any of the tested antimicrobials between the 1997-1998 and 1999-2000 studies. The only change observed was for ampicillin, which had an increase in $\mathrm{MIC}_{90}$ from $2 \mu \mathrm{g} / \mathrm{ml}$ in $1997-1998$ to $8 \mu \mathrm{g} / \mathrm{ml}$ in $1999-2000$. Levofloxacin also displayed potent activity against all isolates $\left(\mathrm{MIC}_{90}, 0.015 \mu \mathrm{g} / \mathrm{ml}\right)$ including the BLNAR isolate (MIC, $0.015 \mu \mathrm{g} / \mathrm{ml}$ ). Although the MIC for levofloxacin ranged from $\leq 0.004 \mu \mathrm{g} / \mathrm{ml}$ to $0.25 \mu \mathrm{g} / \mathrm{ml}$, only two isolates had MICs of 0.25 $\mu \mathrm{g} / \mathrm{ml}$. The activity of levofloxacin was not compromised by $\beta$-lactamase production $\left(\mathrm{MIC}_{90}\right.$, $0.015 \mu \mathrm{g} / \mathrm{ml}$ for both $\beta$-lactamase-positive andnegative isolates).
M. catarrhalis. The susceptibility results for the 49 isolates of $M$. catarrhalis collected during 1999-2000 are shown in Table 4 . $\beta$-lactamase production was detected in $48(98.0 \%)$ of the 49 isolates. The most active agents tested were azithromycin $\left(\mathrm{MIC}_{90}, \leq 0.03\right.$ $\mu \mathrm{g} / \mathrm{ml}$ ) and levofloxacin $\left(\mathrm{MIC}_{90}, 0.03 \mu \mathrm{g} / \mathrm{ml}\right)$. The results in Table 2 show no major changes in the susceptibility of $M$. catarrhalis to the tested antimicrobials during the two seasons, except for decreases in $\mathrm{MIC}_{90} \mathrm{~s}$ for ampicillin (from $>8 \mu \mathrm{g} / \mathrm{ml}$ in $1997-1998$ to $4 \mu \mathrm{g} / \mathrm{ml}$ in 1999-2000) and levofloxacin (from $0.06 \mu \mathrm{g} / \mathrm{ml}$ in $1997-1998$ to $0.03 \mu \mathrm{g} / \mathrm{ml}$ in 1999 2000).

\section{Discussion}

The activity of levofloxacin against key respiratory pathogens was first benchmarked in Brazil in a study we conducted during the 1997-1998 season [12]. In an attempt to monitor any changes in susceptibility of these pathogens to levofloxacin and comparator agents, the current study was conducted during the 1999-2000 season. All five laboratories that participated in the 1997-1998 study also participated in the 1999-2000 study, enabling longitudinal analysis of the resulting antimicrobial susceptibility data. Both studies were also comparable in terms of the numbers of organisms collected and tested. Together, these studies represent one of the most extensive efforts to monitor antimicrobial resistance in respiratory pathogens in Brazil.

Penicillin is widely regarded as a marker for monitoring resistance in pneumococci and is used to stratify the susceptibility of organisms to other classes of antimicrobial agents. However, it was not long ago that penicillin was the therapy of choice for pneumococcal infections because of its efficacy and low cost. The utility of penicillin as a therapeutic agent has been threatened by increasing resistance, beginning with the appearance of the first resistant isolate in Brazil in 1988 [3]. Until recently, very few published studies had been conducted in Brazil to monitor the susceptibility of respiratory pathogens to penicillin and other key agents. Most early studies included Brazil as one 
Table 3. Susceptibility of 274 isolates of $H$. influenzae collected during 1999-2000 to all antimicrobials tested according to $\beta$-lactamase status ${ }^{\mathrm{a}}$

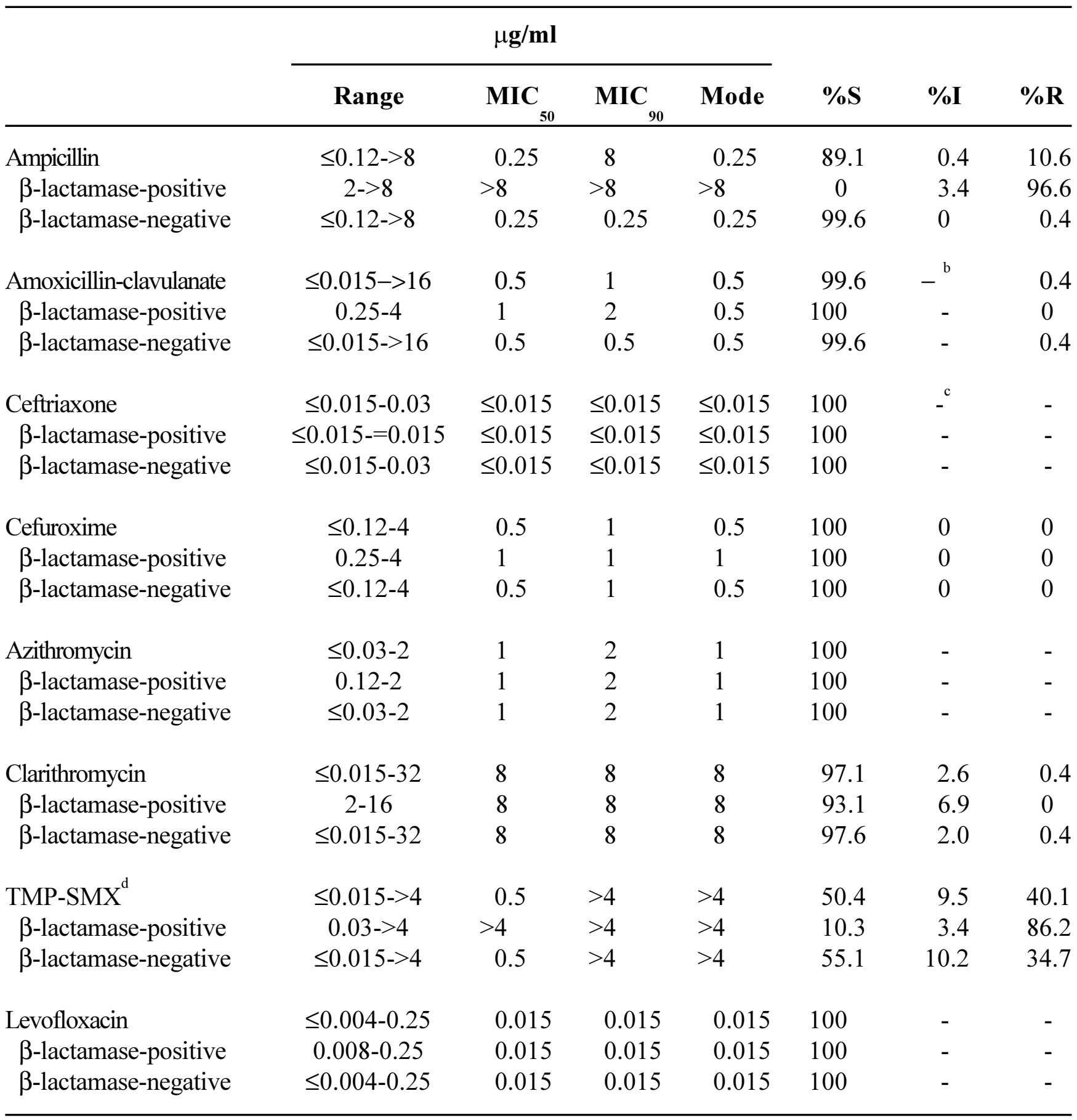

a29 $\beta$-lactamase-positive and $245 \beta$-lactamase-negative $H$. influenzae were collected.

${ }^{b}$ NCCLS breakpoints were unavailable for categorical interpretation as intermediate (I).

'NCCLS breakpoints unavailable for categorical interpretation as intermediate (I) or resistant (R).

dTMP-SMX = trimethoprim-sulfamethoxazole. 
Table 4. Susceptibility of 49 isolates of M. catarrhalis collected during 1999-2000 to all antimicrobials ${ }^{\mathrm{a}}$

\begin{tabular}{lcccc}
\hline & \multicolumn{4}{c}{$\mu \mathbf{g} / \mathbf{m l}$} \\
\cline { 2 - 5 } & Range & MIC $_{\mathbf{5 0}}$ & $\mathbf{M I C}_{\mathbf{9 0}}$ & Mode \\
\hline Ampicillin & $\leq 0.12-4$ & 1 & 4 & 1 \\
Amoxicillin-clavulanate & $\leq 0.015-0.5$ & 0.06 & 0.25 & 0.03 \\
Ceftriaxone & $\leq 0.015-1$ & 0.06 & 0.5 & $\leq 0.015$ \\
Cefuroxime & $0.5-4$ & 1 & 2 & 1 \\
Azithromycin & $\leq 0.03-0.06$ & $\leq 0.03$ & $\leq 0.03$ & $\leq 0.03$ \\
Clarithromycin & $0.06-2$ & 0.12 & 0.12 & 0.12 \\
TMP-SMX & $\leq 0.015-2$ & 0.25 & 0.5 & 0.25 \\
Levofloxacin & $0.015-0.06$ & 0.03 & 0.03 & 0.03 \\
\hline
\end{tabular}

a48 $\beta$-lactamase positive and $1 \beta$-lactamase negative $M$. catarrhalis were collected.

${ }^{\mathrm{b}} \mathrm{TMP}-\mathrm{SMX}=$ trimethoprim-sulfamethoxazole.

component of a larger multi-country study, which resulted in a relatively small number of isolates from Brazil being included in the analysis [11,15-18]. One of the earliest studies to monitor pneumococcal susceptibility in Brazil [19] reported that $81.3 \%$ of 262 isolates collected in Brazil between 1988 and 1992 were susceptible to penicillin. The current report found the susceptibility of $S$. pneumoniae to penicillin unchanged between 1997-1998 and 1999-2000, with $77.1 \%$ to $77.2 \%$ of isolates susceptible, suggesting that penicillin susceptibility in Brazil has remained essentially unchanged in the last decade. Penicillin susceptibility rates in Brazil were slightly lower $(71.9 \%)$ in the SENTRY study of 1997-1998 [20]. The SENTRY study also found that isolates from outpatients were only $59.6 \%$ susceptible [21]. In contrast, our 1999 2000 study found $75.3 \%$ of outpatient isolates to be penicillin-susceptible (data not shown). It is also interesting to note that the current study found a decrease in penicillin-resistant isolates between 19971998 (4.2\%), and 1999-2000 (2.9\%) (Table 2). This is in contrast to findings from the United States, where penicillin resistance increased from $12.3 \%$ in $1997-$ 1998 , to $14.7 \%$ in $1998-1999$ [8].
Penicillin resistance had considerable impact on the susceptibility of $S$. pneumoniae to other $\beta$-lactams, including the cephalosporins. For example, all 13 cefuroxime-resistant isolates in the 1999-2000 study were also penicillin-resistant, and trimethoprimsulfamethoxazole resistance rates were $28 \%, 71 \%$, and $100 \%$ among penicillin-susceptible, -intermediate, and -resistant isolates, respectively. Almost identical results were obtained in the study by Ko, et al. [22], which found trimethoprim-sulfamethoxazole resistance rates of $28 \%$ for penicillin-susceptible, and $76 \%$ for penicillin-intermediate isolates.

The $15 \%$ increase in resistance of $S$. pneumoniae to trimethoprim-sulfamethoxazole between 1997-1998 and 1999-2000, may be due to the extensive use of this agent for respiratory tract infections in the outpatient setting [22]. Other studies have shown that resistance to trimethoprim-sulfamethoxazole in Brazil has risen considerably during the last decade, from $1 \%$ in a 1981 1982 study [23] to $29.2 \%$ in a study conducted between 1988 and 1992 [19]. The widespread use of trimethoprim-sulfamethoxazole may also be exerting selective pressure for penicillin-nonsusceptible phenotypes (serotype 14 clones) [22]. In our 1999- 
2000 study, all penicillin-resistant isolates of $S$. pneumoniae were also resistant to trimethoprimsulfamethoxazole, confirming the observations of other investigators $[18,24,25]$. The increase in trimethoprimsulfamethoxazole resistance is not unique to Brazil; an increase from $13.8 \%$ in $1997-1998$ to $27.3 \%$ in 1998 1999 has also been reported in the United States [8].

To date, few studies have been conducted to compare the efficacy of fluoroquinolones against respiratory pathogens in Brazil [20,26]. In our 19971998 study, only one levofloxacin-resistant isolate was detected out of 359 isolates tested, and no resistant isolates were identified among the 448 isolates tested in 1999-2000. In both studies, levofloxacin had an $\mathrm{MIC}_{90}$ of $1 \mu \mathrm{g} / \mathrm{ml}$, indicating that in $S$. pneumoniae, resistance to levofloxacin has remained unchanged during the previous 2 years and still shows no crossresistance with other classes of antimicrobial agents. Although fluoroquinolone-resistant pneumococci are being reported in small numbers in other countries such as Canada [9] and the United States [8], they have not been detected to any significant extent in Brazil.

Among $H$. influenzae, activity remained unchanged among the tested antimicrobials between the 19971998 and 1999-2000 respiratory seasons. The percentage of isolates producing $\beta$-lactamase was also unchanged between 1997-1998 and 1999-2000 (10.9\% and $10.6 \%$, respectively) and was similar to the rate of $11.8 \%$ reported in the 1997 SENTRY study conducted in Brazil [21]. In a separate study, Casagrande, et al. [27] found that $13.8 \%$ of 391 isolates collected from meningitis patients aged 5 years or less between 1989 and 1995, were positive for the production of $\beta$-lactamase. It was noted that there was no major change in the ampicillin resistance rate, which was $9.6 \%$ in $1997-1998$, and $10.6 \%$ in $1999-2000$; however, there was a four-fold increase in the $\mathrm{MIC}_{90}$, from $2 \mu \mathrm{g} / \mathrm{ml}$ in $1997-1998$ to $8 \mu \mathrm{g} / \mathrm{ml}$ in $1999-2000$. Susceptibility of $H$. influenzae to ampicillin should be carefully monitored in future studies to determine if this shift in MIC distribution continues. The BLNAR isolate that was identified in the current 1999-2000 study was unique; none of the other studies conducted in Brazil have reported isolates with this phenotype.
The ability of $M$. catarrhalis to produce $\beta$ lactamase remained unchanged between 1997-1998 (97.9\%) and 1999-2000 (98.0\%). This rate of $\beta$ lactamase production was slightly higher than the $89.0 \%$ reported in the 1997 SENTRY study [21] and the $91.6 \%$ reported in the Alexander Project [16], which combined Brazilian isolates with those from other countries. The current study found little or no change in the activity of any of the tested agents between the two respiratory seasons based on the $\mathrm{MIC}_{90}$ values. The only exceptions were ampicillin and levofloxacin: the ampicillin $\mathrm{MIC}_{90}$ decreased from $>8 \mu \mathrm{g} / \mathrm{ml}$ in $1997-1998$ to $4 \mu \mathrm{g} / \mathrm{ml}$ in 1999 2000; while the levofloxacin $\mathrm{MIC}_{90}$ decreased from $0.06 \mu \mathrm{g} / \mathrm{ml}$ in $1997-1998$ to $0.03 \mu \mathrm{g} / \mathrm{ml}$ in 1999-2000.

In conclusion, this longitudinal study has shown that, with one exception, there were no major changes in the susceptibility of $S$. pneumoniae, $H$. influenzae, and M. catarrhalis to commonly tested and prescribed antimicrobial agents between the 1997-1998 and 1999-2000 respiratory seasons in Brazil. The exception was $S$. pneumoniae and trimethoprim-sulfamethoxazole, for which resistance increased from $23.4 \%$ in $1997-1998$, to $38.6 \%$ in 1999-2000. The design of this study and our previous 1997-1998 study, with isolates collected in similar numbers and from the same laboratories over two successive respiratory seasons, provided a unique opportunity to monitor any changes in antimicrobial susceptibility profiles among three important respiratory pathogens. Similar ongoing surveillance initiatives in various global regions will be crucial to maintain an accurate picture of antimicrobial resistance in Brazil and other countries.

\section{Acknowledgements}

Focus Technologies, Inc., would like to gratefully acknowledge Daiichi Pharmaceutical Co., Ltd., for funding this study and thank each of the participating laboratories and their staffs for their cooperation in conducting this study. 


\section{References}

1. Hansman D., Bullen M. M. A resistant pneumococcus. Lancet ii 1967;246-65.

2. Appelbaum, P. C. Antimicrobial resistance in Streptococcus pneumoniae: an overview. Clin Infect Dis 1992; 15:77-83.

3. De Sousa Marques H. H., Yamamoto M., Sakane P. T., et al. Relatively penicillin-resistant pneumococcal meningitis in a Brazilian infant. Pediatr Infect Dis J 1988;7:433-4.

4. Breiman R. F., Butler J. C., Tenover F. C., et al. Emergence of drug resistant pneumococcal infections in the United States. J Am Med Assoc 1994;271:1831-5.

5. Butler J. C., Hofmann J., Cetron M. S., et al. The continued emergence of drug resistant Streptococcus pneumoniae in the United States: an update from the Centers for Disease Control and Prevention's Pneumococcal Sentinel Surveillance System. J Infect Dis 1996; 174:98693.

6. Thornsberry C., Ogilvie P., Kahn J., Mauriz Y. Surveillance of antimicrobial resistance in Streptococcus pneumoniae, Haemophilus influenzae and Moraxella catarrhalis in the United States in 1996-1997 respiratory season. The Laboratory Investigator Group. Diag Microbiol Infect Dis 1997;29:249-57.

7. Thornsberry C., Sahm D. F. Antimicrobial Resistance in respiratory pathogens: results of an international surveillance study. Chemother 2000;46:15-23.

8. Sahm D.F., Karlowsky J. A., Kelly L. J., et al. Need for annual surveillance of antimicrobial resistance in Streptococcus pneumoniae in the United States: 2-Year longitudinal analysis. Antimicrob Agents Chemother 2001;45.

9. Chen D. K., McGreer A., De Azavedo J. C., Low D. E., Decreased susceptibility of Streptococcus pneumoniae to fluoroquinolones in Canada. N Engl J Med 1999;341:233-9.

10. Sahm D. F., Jones M. E., Hickey M. L., et al. Resistance surveillance of Streptococcus pneumoniae, Haemophilus influenzae and Moraxella catarrhalis isolates in Asia and Europe, 1997-1998. J. Antimicrob Chemother 2000;45:457-66.

11. Felmingham D., Washington J. Trends in the antimicrobial susceptibility of bacterial respiratory tract pathogens — findings of the Alexander Project 1992-1996. J Chemother 1999; Suppl1:5-21.

12. Critchley I. A., Thornsberry C., Piazza, et al. Antimicrobial susceptibility of Streptococcus pneumoniae, Haemophilus influenzae and Moraxella catarrhalis collected from five centers in Brazil, 1997-98. Clin Microbiol Infect 2000;6:178-84.
13. National Committee for Clinical Laboratory Standards. Performance Standards for Antimicrobial Susceptibility testing; Seventh Informational Supplement. M100-S7, 1997.

14. National Committee for Clinical Laboratory Standards. Performance Standards for Antimicrobial Susceptibility testing; Ninth Informational Supplement. M100-S9, 1999.

15. Jacobs M. R., Appelbaum P. C. Susceptibility of 1,100 Streptococcus pneumoniae isolates isolated in 1997 from seven Latin American and Caribbean countries. Laser Study Group. Int J Antimicrob Agents 2000;16:17-24.

16. Felmingham D., Gruneberg R. N., The Alexander Project 1996-1997: latest susceptibility data from this international study of bacterial pathogens from community-acquired lower respiratory tract infections. J Antimicrob Chemother 2000;45:191-203.

17. G. O. C., Soto-Hernandez J. L. In vitro activity of moxifloxacin (BAY 12-8039) against respiratory tract pathogens from six Latin American countries. Chemotherapy 2000;46:379-82.

18. Tomasz A., Corso A., Severina E. P., et al. Molecular epidemiologic characterization of penicillin-resistant Streptococcus pneumoniae invasive pediatric isolates recovered in six Latin-American countries: an overview. Microb Drug Resist 1998;4:195-207.

19. Sessegolo J. F., Levin A. S., Levy C. E., et al. Distribution of serotypes and antimicrobial resistance of Streptococcus pneumoniae isolates isolated in Brazil from 1998 to 1992. J Clin Microbiol 1994;32:906-11.

20. Sader H. S., Gales A. C., Granacher T. D., et al. Prevalence of antimicrobial resistance among respiratory tract isolates in Latin America: Results from SENTRY antimicrobial surveillance program (1997-1998). Braz J Infect Dis 2000;4:245-54.

21. Sader H. S., Sampaio L.M., Zoccoli C., Jones R. N. Results of the 1997 SENTRY Antimicrobial Surveillance Program in Three Brazilian Medical Centers. Braz J Infect Dis 1999;3:63-79.

22. Ko A. I., Reis J. N., Coppola S. J., et al. Clonally related penicillin-nonsusceptible Streptococcus pneumoniae serotype 14 from cases of meningitis in Salvador, Brazil, Clin Infect Dis 2000;30:78-86.

23. Teixeira L. M., Andrade J. R. C., Lourenco N. J. Serotypes and antimicrobial susceptibility of Streptococcus pneumoniae isolates in Rio de Janeiro, Brazil. Rev Microbiol Sao Paulo 1988; 19:93-4.

24. De Cunto Brandileone M. C., Vieira S. D., Tadeu Casagrande S., et al. Characteristics of isolates of Streptococcus pneumoniae from middle-aged and elderly patients in Brazil: capsular serotypes and antimicrobial sensitivity to invasive infections. Braz $\mathrm{J}$ Infect Dis 1998;2:90-6. 
25. Levin A. S., Teixeira L. M., Sessegolo J. F., Barone A. A. Resistance of Streptococcus pneumoniae to antimicrobials in Sao Paulo, Brazil: clinical features and serotypes. Rev Inst Med Trop Sao Paulo 1996;38:187-92.

26. Mendes C., Sader H., Dainesi S. M., et al. Evaluation of the antimicrobial activity of sparfloxacin, relative to other oral antibiotics against 1,125 bacterial isolates from 10 medical centers in Brazil. Braz J Infect Dis 1998; 2:18-24.

27. Casagrande S. T., Vicente E. J., Landgraf I. M., Kobata A. M. Antimicrobial resistance patterns of Haemophilus influenzae isolated from patients with meningitis in Sao Paulo, Brazil. Braz J Med Biol Res 2000;33:295-300. 